Conditional symmetries for boundary value problems: new definition and its application for nonlinear problems with Neumann conditions

\author{
Roman Cherniha
}




\title{
CONDITIONAL SYMMETRIES FOR BOUNDARY VALUE PROBLEMS: NEW DEFINITION AND ITS APPLICATION FOR NONLINEAR PROBLEMS WITH NEUMANN CONDITIONS
}

\author{
ROMAN CHERNIHA
}

\begin{abstract}
A new definition of conditional invariance for boundary-value problems (BVPs) is proposed. Its relationship with the definitions given earlier for Lie's invariance, is shown and a wider applicability is demonstrated via a non-trivial example.The definition is applied to reduction of BVPs with the basic reaction-diffusion-convection equation, which is used in physically and biologically motivated problems. The constrains on the functions arising in the boundary conditions are established and the nonlinear BVPs in question are thus reduced to those based on linear second-order ODEs. Moreover, the exact solution of a nonlinear BVP with zero Neumann conditions is found.
\end{abstract}

2000 Mathematics Subject Classification: 22E70, 35K60, 35K57

Keywords: Lie symmetry, $Q$-conditional symmetry, boundary-value problem, exact solution

\section{INTRODUCTION}

Nowadays Lie symmetries are widely applied to partial differential equations (including multi-component systems of multidimensional PDEs), notably, to their reductions to ordinary differential equations (ODEs) and constructing exact solutions. There are a huge number of papers and many excellent books (see, e. g., $[3,4,12$, $18,19]$ and papers cited therein) devoted to such applications. Over recent decades, other symmetry methods based on the classical Lie-Ovsiannikov method have been derived. The Bluman-Cole method of non-classical symmetry (other widely used terminology is $Q$-conditional symmetry) is the most noted among them and [3] summarizes the results obtained by means of this approach for scalar PDEs while in recent papers $[6,7]$ this method is applied to non-linear PDE systems. Further generalizations of the classical Lie-Ovsiannikov method were introduced by many authors (see, e. g., [11] for details).

However, a PDE (a system of PDEs) cannot model any real process without additional condition(s) for the unknown function(s). On the other hand, the relevant boundary-value problem (BVP) based on the given PDE (system of PDEs) can describe many real processes arising in nature and society. One may note that the symmetry-based methods were not widely used for solving BVPs. The obvious 
reason follows from the following observation: the relevant boundary and initial conditions are usually not invariant under any transformations, i. e., they don't admit any symmetry of the governing PDE(s). Nevertheless, there are some classes of BVPs which can be solved by means of the Lie symmetry based algorithm. This algorithm uses the notion of Lie's invariance of BVP in question. Probably, the first rigorous definition of Lie's invariance for BVPs was formulated by G.W. Bluman in 1970s [2] (the definition and several examples are summarized in the book [3]). This definition was used (explicitly or implicitly) in several papers to derive exact solutions of some BVPs. It should be noted that Ibragimov's definition of BVP invariance [14] (see also his recent paper [15]), which was formulated independently, is equivalent to Bluman's. On the other hand, one notes that Bluman's definition is not suitable for all types of boundary conditions. Notably, the definition doesn't work in the case of boundary conditions involving points at infinity (e. g., $x \rightarrow \infty$ ) and conditions on the moving surfaces (e. g., the Stefan conditions). J. R. King was probably the first to note the problem with points at infinity and suggested the appropriate substitution to transform such points to regular those [16]. In [20] (several examples are summarized in [1]), the way was proposed of defining Lie's invariance on the moving boundaries in the case of some BVPs arising in hydrodynamics. In our recent papers [8-10], a new definition of Lie's invariance of BVP with a wide range of boundary conditions (including those on non-regular manifolds and moving surfaces) was formulated in two-dimensional and multidimensional cases, respectively. Moreover, an algorithm of the group classification for the given class of BVPs was created for the first time. The definition and algorithm were applied to a class of nonlinear two-dimensional and multidimensional BVPs of Stefan type with the aim to show their efficiency. However, there are many realistic BVPs that cannot be solved using any definition of Lie's invariance of BVP (a simple example will be presented in the next section), hence, definitions involving more general types of symmetries should be proposed.

In section 2, new definition of conditional invariance of BVPs with a wide range of boundary conditions is presented. The known earlier definitions of Lie's invariance follow as particular cases from this definition but not vice versa. In section 3, to demonstrate the efficiency of the definition, BVPs based on the widely used nonlinear reaction-diffusion-convection equations with the Neumann boundary conditions are examined. Reductions of the BVPs in question with correctly-specified boundary conditions to BVPs for ODEs are obtained and examples of exact solutions are presented. As a conclusion, we summarize the results obtained.

\section{LIE'S AND CONDITIONAL INVARIANCE OF BVPS}

First of all, to avoid possible complications we restrict ourselves to the case when the basic equation of BVP is a two-dimensional evolution PDE of $k$ th-order $(k \geq 2)$. 
In this case, the relevant BVP may be formulated as follows:

$$
\begin{gathered}
u_{t}=F\left(x, u, u_{x}, \ldots, u_{x}^{(k)}\right),(t, x) \in \Omega \subset \mathbb{R}^{2} \\
s_{a}(t, x)=0: B_{a}\left(t, x, u, u_{x}, \ldots, u_{x}^{\left(k_{a}\right)}\right)=0, a=1,2, \ldots, p, k_{a}<k
\end{gathered}
$$

where $F$ and $B_{a}$ are smooth functions in the corresponding domains, $\Omega$ is a domain with smooth boundaries and $s_{a}(t, x)$ are smooth curves. Hereafter the subscripts $t$ and $x$ denote differentiation with respect to these variables, $u_{x}^{(j)}=\frac{\partial^{j} u}{\partial x^{j}}, j=$ $1,2, \ldots, k$. We assume that BVP (2.1)-(2.2) has a classical solution (in the usual sense).

Consider the infinitesimal generator

$$
X=\xi^{0}(t, x) \frac{\partial}{\partial t}+\xi^{1}(t, x) \frac{\partial}{\partial x}+\eta(t, x, u) \frac{\partial}{\partial u}
$$

Hereafter, $\xi^{0}, \xi^{1}$ and $\eta$ are known smooth functions. Let us assume that this operator defines a Lie symmetry acting on both $(t, x, u)$-space as well as on its projection to $(t, x)$-space. Let $\underset{k}{X}$ be the $k$ th prolongation of the generator $X$ calculated by the well-known prolongation formulae (see, e.g. $[18,19])$.

Definition 1. [4] The Lie symmetry $X(2.3)$ is admitted by the boundary value problem (2.1)-(2.2) if and only if:

(a) $\underset{k}{X}\left(F\left(x, u, u_{x}, \ldots, u_{x}^{(k)}\right)-u_{t}\right)=0$ when $u$ satisfies (2.1);

(b) $X\left(s_{a}(t, x)\right)=0$ when $s_{a}(t, x)=0, a=1, \ldots, p$;

(c) $\underset{k_{a}}{X}\left(B_{a}\left(t, x, u, u_{x}, \ldots, u_{x}^{\left(k_{a}\right)}\right)\right)=0$ when $\left.B_{a}\right|_{s_{a}(t, x)=0}=0, a=1, \ldots, p$.

Because BVP (2.1)-(2.2) involves only the simplest boundary conditions, Definition 1 cannot be applied to BVP with boundary conditions defined on the non-regular manifolds and those on the unknown moving surfaces. In the recent papers $[8,9]$, it was shown how this definition can be be extended to the classes of BVPs with more complicated boundary and initial conditions. Nevertheless, the relevant definitions coincide with one another if the given BVP in based on the two-dimensional PDE of the form (2.1), here the definition from paper [9] will be used below for further generalization.

Consider a BVP for the evolution equation (2.1) involving conditions (2.2) and some other types of the boundary and initial conditions, namely:

$$
S(t, x)=0: B^{l}\left(t, x, u, \ldots, u_{x}^{\left(k_{l}\right)}, S, \frac{\partial S}{\partial t}, \frac{\partial S}{\partial x}\right)=0, l=1, \ldots, n_{1},
$$

and

$$
\gamma_{c}(t, x)=\infty: \Gamma_{c}\left(t, x, u, u_{x}, \ldots, u_{x}^{\left(k_{c}\right)}\right)=0, c=1,2, \ldots, n_{2} .
$$


Here $k_{l}<k, k_{c}<k, n_{1}$ and $n_{2}$ are the given numbers, $\gamma_{c}(t, x)$ are the known functions, while the function $S(t, x)$ defining free boundary surface must be found (in contrast to the known functions $s_{a}(t, x)$ in (2.2)!). We assume that all functions arising in (2.1)-(2.2) and (2.4)-(2.5) and the number of boundary and initial conditions are such that the classical solution exists for this BVP.

Let us assume that the operator

$$
Q=\xi^{0}(t, x, u) \frac{\partial}{\partial t}+\xi^{1}(t, x, u) \frac{\partial}{\partial x}+\eta(t, x, u) \frac{\partial}{\partial u}
$$

is a $Q$-conditional symmetry of $\operatorname{PDE}(2.2)$, i. e., the following well-known criterion is satisfied

$$
\left.\underset{k}{Q F} \equiv \underset{k}{Q}\left(u_{t}-F\left(t, x, u, u_{x}, \ldots, u_{x}^{(k)}\right)\right)\right|_{M}=0,
$$

where $\underset{k}{Q}$ is the $k$ th prolongation of $Q$ and the manifold $M=\{F=0, Q(u) \equiv$ $\left.\xi^{0}(t, x) u_{t}+\xi^{1}(t, x) u_{x}-\eta(t, x, u)=0\right\}$.

Definition 2. BVP (2.1)-(2.2) and (2.4)-(2.5) is $Q$-conditionally invariant under operator (2.6) if:

(a) the criterion (2.7) is fulfilled;

(b) $Q\left(s_{a}(t, x)\right)=0$ when $s_{a}(t, x)=0,\left.\quad B_{a}\right|_{s_{a}(t, x)=0}=0, a=1, \ldots, p$;

(c) $\underset{k_{a}}{Q}\left(B_{a}\left(t, x, u, u_{x}, \ldots, u_{x}^{\left(k_{a}\right)}\right)\right)=0$ when $\left.B_{a}\right|_{s_{a}(t, x)=0}=0, a=1, \ldots, p$;

(d) $\underset{k_{l}}{Q}\left(B^{l}\left(t, x, u, \ldots, u_{x}^{\left(k_{l}\right)}, S, \frac{\partial S}{\partial t}, \frac{\partial S}{\partial x}\right)\right)=0$ when $\left.B^{l}\right|_{S(t, x)=0}=0, l=1, \ldots, n_{1}$;

(e) $Q_{*}\left(\gamma_{c}^{*}(\tau, y)\right)=0$ when $\gamma_{c}^{*}(\tau, y)=0, c=1,2, \ldots, n_{2}$;

(f) $\underset{k_{c}}{Q_{*}}\left(\Gamma_{c}^{*}\left(\tau, y, u, u_{y}, \ldots, u_{y}^{\left(k_{c}\right)}\right)\right)=0$ when $\left.\Gamma_{c}^{*}\right|_{\gamma_{c}^{*}(\tau, y)=0}=0, c=1, \ldots, n_{2}$,

where $Q_{*}, \Gamma_{c}^{*}$ and $\gamma_{c}^{*}(\tau, y)$ are operators (2.6), the functions $\Gamma_{c}$ and $\frac{1}{\gamma_{c}(t, x)}$, respectively, expressed via the new independent variables

$$
\tau=\left\{\begin{array}{ll}
t, & \text { if } \frac{\partial \gamma_{c}(t, x)}{\partial x} \neq 0, \\
\frac{1}{\gamma_{c}(t, x)}, & \text { if } \frac{\partial \gamma_{c}(t, x)}{\partial x}=0
\end{array} \quad y= \begin{cases}x, & \text { if } \frac{\partial \gamma_{c}(t, x)}{\partial x}=0, \\
\frac{1}{\gamma_{c}(t, x)}, & \text { if } \frac{\partial \gamma_{c}(t, x)}{\partial x} \neq 0 .\end{cases}\right.
$$

Remark 1. In item (f) of Definition 2, we take into account that any physically motivated boundary condition (2.5) contains the expression $\gamma_{c}(t, x)=\infty$, which is equivalent either to $\gamma_{c}^{0}(t)=\infty$ or to $\gamma_{c}^{1}(x)=\infty$. It means that the form of the substitution does not depend on the choice of partial derivatives in (2.8) because the assumption $\frac{\partial \gamma_{c}(t, x)}{\partial x} \neq 0$ automatically leads to $\frac{\partial \gamma_{c}(t, x)}{\partial t}=0$ and vice versa.

It should be noted that this definition coincides with Definition 1 if the operator (2.6) is simultaneously Lie's symmetry (2.3) and there are no boundary conditions of the form (2.4) and (2.5). 
Nevertheless, Definition 2 seems to be a direct generalization of the definition from $[9$, p. 98] , such generalization makes it possible to extend essentially the applicability of symmetries for BVPs. A simple example is presented below.

Example 1. Consider the reaction-diffusion-convection equation

$$
u_{t}=u_{x x}+\lambda u u_{x}+u(1-u) .
$$

where $\lambda$ is an arbitrary non-zero constant. Equation (2.9) was introduced in [17] as a generalization of the well-known Fisher equation (i. e., (2.9) with $\lambda=0$ ) by adding the simple convection term, therefore, being named the Murray equation. As follows from [5], this equation is invariant with respect to the trivial Lie algebra spanned by the operators $P_{t}=\partial / \partial t$ and $P_{x}=\partial / \partial x$. Let us consider the BVP on the semi-infinite space interval $(0 ;+\infty)$ for the Murray equation (2.9) with boundary conditions

$$
x=0: u=\varphi_{1}(t), \quad x=\infty: u=\varphi_{2}(t),
$$

where $\varphi_{1}(t), \varphi_{2}(t)$ are arbitrary smooth functions.

Let us apply items (b) and (c) from Definition 1 to a linear combination of the operators $P_{t}$ and $P_{x}$, say $X=\lambda_{0} P_{t}+\lambda_{1} P_{x}$. One easily checks that item (b) immediately leads to the restriction $\lambda_{1}=0$, i. e., the BVP in question can be invariant only under the operator $P_{t}$ generating the one-parameter time translation group. Now we apply item (c) to the first boundary condition from (2.10) and obtain the restriction $\varphi_{1}^{\prime}(t)=0$. One also notes by direct checking that the second boundary condition from (2.10) is invariant under time translation provided $\varphi_{2}^{\prime}(t)=0$. It means that BVP (2.9)-(2.10) is not invariant under any linear combination of the operators $P_{t}$ and $P_{x}$, excluding a special case when both functions $\varphi_{1}(t)$ and $\varphi_{2}(t)$ are constants. Moreover, in this special case, the invariant solution of this problem must be stationary (the operator $P_{t}$ generates such solutions only), i. e., the result is rather trivial.

It turns out that BVP (2.9)-(2.10) with arbitrary smooth functions $\varphi_{1}(t)$ and $\varphi_{2}(t)$ is $Q$-conditionally invariant according to Definition 2. In fact, equation (2.9) admits the $Q$-conditional symmetry [5]

$$
Q=\partial_{t}-\left(\lambda u+\frac{1}{\lambda}\right) \partial_{x}+\left(u-u^{2}\right) \partial_{u}
$$

Because each conditional symmetry can be multiplied by an arbitrary smooth function, for instance $f(x)$, this operator can be rewritten as

$$
Q=f(x) \partial_{t}-f(x)\left(\lambda u+\frac{1}{\lambda}\right) \partial_{x}+f(x)\left(u-u^{2}\right) \partial_{u} .
$$

Now one sees that item (a) from Definition 2 is fulfilled by the correct choice of the operator $Q$, while the first boundary condition from (2.10) satisfies items (b) and (c) provided $f(0)=0$. Note that there is no need to calculate any prolongation of the operator $Q$ in this case because conditions (2.10) do not involve derivatives of $u$. Finally, we check whether items (e) and (f) are fulfilled because the second boundary 
condition from (2.10) involves a non-regular manifold (item (d) is not applicable for BVP in question). Applying formulae (2.8) to operator (2.12), one obtains

$$
Q_{*}=f(1 / y) \partial_{t}+f(1 / y) y^{2}\left(\lambda u+\frac{1}{\lambda}\right) \partial_{y}+f(1 / y)\left(u-u^{2}\right) \partial_{u} .
$$

Simultaneously, the relevant boundary condition takes the form

$$
y=0: u=\varphi_{2}(t) .
$$

So, items (e) and (f) are fulfilled provided $f(1 / y) \rightarrow 0$ if $y \rightarrow 0$, i. e. $f(x) \rightarrow 0$ if $x \rightarrow \infty$.

Thus, BVP (2.9)-(2.10) is $Q$-conditionally invariant under operator (2.12) if $f(0)=$ 0 and $f(x) \rightarrow 0$ if $x \rightarrow \infty$ (in particular, one may set $f(x)=x e^{-x}$ ).

\section{CONDITIONAL INVARIANCE AND EXACT SOLUTIONS OF SOME NON-LINEAR BVPS}

Here we consider some BVPs with the basic reaction-diffusion-convection equation

$$
u_{t}=\left[u^{m} u_{x}\right]_{x}+\lambda u^{m} u_{x}+\left(\lambda_{1} u^{m+1}+\lambda_{2}\right)\left(u^{-m}-\lambda_{3}\right),
$$

where $\lambda_{k}, k=1,2,3$ and $m \neq-1,0$ are arbitrary constants. This equation with correctly-specified coefficients generates several equations, which are used in physically and biologically motivated problems (see examples in [11]). Let us formulate a BVP with the governing equation in the domain $\Omega=\{(t, x): t>0, x \in$ $\left.\left(x_{1}, x_{2}\right), x_{1}<x_{2} \in \mathbb{R}\right\}$ using the initial profile

$$
\begin{gathered}
t=0: u=\varphi_{0}(x) \\
x=x_{a}: u_{x}=\varphi_{a}(t), a=1,2,
\end{gathered}
$$

where $\varphi_{0}(x), \varphi_{a}(t)$ are the known smooth functions. So, (3.1)-(3.3) is the nonlinear BVP, which is the known subject for investigation in pure and applied mathematics. In [11], it was proved that (3.1) admits the $Q$-conditional symmetry

$$
Q=\partial_{t}+\left(\lambda_{1} u+\lambda_{2} u^{-m}\right) \partial_{u},
$$

which is not equivalent to any Lie's symmetry provided $\lambda_{2} \neq 0$. Because each conditional symmetry can be multiplied by an arbitrary smooth function, for instance $f(t)$, the operator

$$
Q=f(t) \partial_{t}+f(t)\left(\lambda_{1} u+\lambda_{2} u^{-m}\right) \partial_{u}
$$

will be examined instead of (3.5).

Now we apply Definition 2 to BVP (3.1)-(3.3) in order to obtain the correctlyspecified constraints when this problem is conditionally invariant under operator (3.5). Obviously, the first item is fulfilled by the correct choice of the operator. Item (b) is satisfied provided $f(0)=0$ which leads to the restriction on the operator.

A non-trivial result is obtained by applying item (c). For the initial profile the condition is automatically fulfilled because $Q\left(u-\varphi_{0}(x)\right) \equiv 0$ if $f(0)=0$. However, 
the story is different with the boundary conditions (3.3). In fact, calculating the first prolongation of operator (3.5) according to the item (c) with $k_{a}=1$ and acting on $u_{x}=\varphi_{a}(t)$, one obtains two first-order ODEs

$$
\varphi_{a}(t)\left(\lambda_{1}-m \lambda_{2}\left(u\left(t, x_{a}\right)\right)^{-m-1}\right)=\dot{\varphi}_{a}(t), a=1,2 .
$$

to find the functions $\varphi_{a}(t)$. Because the BVP in question does not involve any conditions of the form (2.4)-(2.5), there is no need to examine items (d)-(f). Thus, we obtain

Proposition 1. $B V P$ (3.1)-(3.3) is $Q$-conditionally invariant under operator (3.5) if and only if $f(0)=0$ and constraints (3.6) hold.

One may note that constraints (3.6) are two additional boundary conditions because they involve the function $u(t, x)$. Hence, it may lead to the non-existence of a classical solution to the BVP in question. Fortunately, there are cases when constrains (3.6) do not produce any boundary conditions:

(i) $\varphi_{a}(t)=0, a=1,2$;

(ii) $\lambda_{2}=0$.

In case (i), BVP with the zero Neumann conditions

$$
x=x_{a}: u_{x}=0, a=1,2,
$$

are obtained. Such conditions (zero flux on boundaries) are widely used in physical and biological problems.

In case (ii), BVP takes the form

$$
\begin{gathered}
u_{t}=\left[u^{m} u_{x}\right]_{x}+\lambda u^{m} u_{x}+\lambda_{1} u\left(1-\lambda_{3} u^{m}\right), \\
x=x_{a}: u_{x}=C_{a} \exp \left(\lambda_{1} t\right), a=1,2,
\end{gathered}
$$

where $C_{a}, a=1,2$ are arbitrary constants, and the initial profile is (3.2).

Now we demonstrate how the result obtained above can be applied to reducing the BVP in question and finding exact solution in case (i). The following ansatz is constructed using the standard procedure, i. e., solving the linear equation $Q(u)=0$ for operator (3.5):

$$
V(t, x) \equiv u^{m+1}= \begin{cases}\lambda_{2}(m+1) t+\varphi(x), & \lambda_{1}=0, \\ \varphi(x) e^{\lambda_{1}(m+1) t}-\frac{\lambda_{2}}{\lambda_{1}}, & \lambda_{1} \neq 0\end{cases}
$$

where $\varphi(x)$ is an unknown function. Substituting (3.10) with $\lambda_{1} \neq 0$ into the govern equation (3.1), one arrives at the ordinary differential equation (ODE)

$$
\varphi_{x x}+\lambda \varphi_{x}-(m+1) \lambda_{1} \lambda_{3} \varphi=0 .
$$

Simultaneously, ansatz (3.10) reduces the boundary conditions (3.7) to the form

$$
x=x_{a}: \varphi_{x}=0, \quad a=1,2 .
$$


Finally, the reduction requires the initial profile to be correctly-specified as follows

$$
t=0: u=\varphi_{0}(x) \equiv\left(\varphi(x)-\frac{\lambda_{2}}{\lambda_{1}}\right)^{\frac{1}{m+1}} .
$$

Thus, the Sturm-Liouville problem (3.11)-(3.12) is obtained to find the function $\varphi(x)$. One easily proves that its non-trivial solution exists only for $\delta=\lambda^{2}+4 \lambda_{1}(m+$ 1) $\lambda_{3}<0$ and a discrete set of values $x_{1}$ and $x_{2}$. To avoid cumbersome calculations, we present the following particular solution of problem (3.11)-(3.12)

$$
\varphi(x)=C_{1} \exp \left(-\frac{\lambda}{2} x\right)\left(\cos \frac{\sqrt{-\delta}}{2} x+\frac{\lambda}{\sqrt{-\delta}} \sin \frac{\sqrt{-\delta}}{2} x\right),
$$

which is valid on the space interval $\left(x_{1}, x_{2}\right)$ with $x_{a}=\frac{2}{\sqrt{-\delta}} \pi n_{a}, n_{a} \in \mathbb{Z}$ and $n_{1}<n_{2}$.

Thus, substituting (3.14) into the second ansatz from (3.10), one immediately arrives at the exact solution

$u=\left(C_{1} \exp \left(-\frac{\lambda}{2} x+\lambda_{1}(m+1) t\right)\left(\cos \frac{\sqrt{-\delta}}{2} x+\frac{\lambda}{\sqrt{-\delta}} \sin \frac{\sqrt{-\delta}}{2} x\right)-\frac{\lambda_{2}}{\lambda_{1}}\right)^{\frac{1}{m+1}}$

of the nonlinear BVP (3.1) with $\lambda_{1} \neq 0,(3.7)$ and (3.13) in the domain $\Omega=\{(t, x)$ : $\left.t>0, x \in\left(x_{1}, x_{2}\right), x_{a}=\frac{2}{\sqrt{-8}} \pi n_{a}, n_{a} \in \mathbb{Z}\right\}$.

Finally, it should be noted that BVP (3.8),(3.9) and (3.2) can be examined in a similar way.

\section{Conclusions}

In this paper, a new definition of conditional invariance for BVPs is proposed. Its relationship with the definitions, given earlier for Lie's invariance, is shown and the applicability of the $Q$-conditional invariance is demonstrated. In particular, the example of BVP, which is $Q$-conditionally invariant under the given operator and is not invariant under any Lie symmetry operator, is presented. The definition is applied to the reduction of BVPs with the basic reaction-diffusion-convection equation (3.1). The constrains on the functions arising in the boundary conditions and allowing the reduction to the linear BVP with the basic second-order ODE are established. Moreover, the exact solution of the nonlinear BVP with zero Neumann conditions is found.

In conclusion, it should be noted that in [13] an algorithm was proposed allowing one to construct so-called side infinitesimals of the form (2.3) for BVP involving two-dimensional evolution equations. We assume that those 'side infinitesimals' are connected with the operators of $Q$-conditional symmetry, however, this is rather difficult to check because the examples presented in [13] are very cumbersome. 


\section{ACKNOWLEDGMENTS}

The author is grateful to the unknown referee for the useful comments. This research was supported by a Marie Curie International Incoming Fellowship within the 7th European Community Framework Programme.

\section{REFERENCES}

[1] V. K. Andreev, O. V. Kaptsov, V. V. Pukhnachov, and A. A. Rodionov, Application of GroupTheoretical Methods in Hydrodynamics. Dordrecht: Kluwer Academic Publishers, 1998.

[2] G. W. Bluman, "Application of the general similarity solution of the heat equation to boundary value problems," Q. Appl. Math., vol. 31, pp. 403-415, 1974.

[3] G. W. Bluman and S. C. Anco, Symmetry and Integration Methods for Differential Equations. New York: Springer, 2002.

[4] G. Bluman and S. Kumei, Symmetries and Differential Equations. Berlin: Springer, 1989.

[5] R. Cherniha, "New $Q$-conditional Symmetries and Exact Solutions of Some Reaction-DiffusionConvection Equations Arising in Mathematical Biology," J. Math. Anal. Appl., vol. 326, pp. 783$799,2007$.

[6] R. Cherniha, "Conditional symmetries for systems of PDEs: new definition and its application for reaction-diffusion systems," J. Phys. A: Math. Theor, vol. 43, p. 405207 (13 pp.), 2010.

[7] R. Cherniha and V. Davydovych, "Lie and conditional symmetries of the three-component diffusive Lotka-Volterra system,” J. Phys. A: Math. Theor., vol. 46, p. 185204 (14 pp.), 2013.

[8] R. Cherniha and S. Kovalenko, "Lie symmetries and reductions of multi-dimensional boundary value problems of the Stefan type," J. Phys. A: Math. Theor., vol. 44, p. 485202 (25 pp.), 2011.

[9] R. Cherniha and S. Kovalenko, "Lie symmetry of a class of nonlinear boundary value problems with free boundaries," Banach Center Publ., vol. 93, pp. 95-104, 2011.

[10] R. Cherniha and S. Kovalenko, "Lie symmetries of nonlinear boundary value problems," Commun. Nonlinear Sci. Numer. Simulat., vol. 17, pp. 71-84, 2012.

[11] R. Cherniha and O. Pliukhin, "New conditional symmetries and exact solutions of nonlinear reaction-diffusion-convection equations," J. Phys. A: Math. Theor., vol. 40, pp. 10049-10070, 2007.

[12] W. I. Fushchych, W. M. Shtelen, and M. I. Serov, Symmetry Analysis and Exact Solutions of Equations of Nonlinear Mathematical Physics. Dordrecht: Kluwer Academic Publishers, 1993.

[13] J. Goard, "Finding symmetries by incorporating initial and side conditions," Euro. J. Appl. Math., vol. 19, pp. 701-715, 2008.

[14] N. H. Ibragimov, "Group analysis of ordinary differential equations and the invariance principle in mathematical physics (on the occasion of the 150th anniversary of the birth of Sophus Lie)," Russ. Math. Surv., vol. 47, pp. 89-156, 1992.

[15] N. H. Ibragimov, "Lie group analysis of Moffat's model in metallurgical industry," J. Nonlinear Math. Phys., vol. 18, pp. 143-162, 2011.

[16] J. R. King, "Exact results for the nonlinear diffusion equations $\frac{\partial u}{\partial t}=\frac{\partial}{\partial x}\left(u^{-\frac{4}{3}} \frac{\partial u}{\partial x}\right)$ and $\frac{\partial u}{\partial t}=$ $\frac{\partial}{\partial x}\left(u^{-\frac{2}{3}} \frac{\partial u}{\partial x}\right)$, , J. Phys. A: Math. Gen., vol. 24, pp. 5721-5745, 1991.

[17] J. D. Murray, Nonlinear Differential Equation Models in Biology. Oxford: Clarendon Press, 1977.

[18] P. J. Olver, Applications of Lie Groups to Differential Equations. New York: Springer, 1993.

[19] L. V. Ovsiannikov, The Group Analysis of Differential Equations. New York: Academic Press, 1982. 
[20] V. V. Pukhnachov, "Invariant solutions of the Navier-Stokes equations describing motion with a free boundary," Dokl. Akad. Nauk SSSR (Rep. Acad. Sci. USSR), vol. 202, pp. 302-305, 1972, (in Russian).

\section{Author's address}

\section{Roman Cherniha}

Institute of Mathematics, Ukrainian National Academy of Sciences, 3 Tereshchenkivs'ka St., 01601 Kyiv, Ukraine

Current address: School of Mathematical Sciences, University of Nottingham, University Park, Nottingham, NG7 2RD, UK

E-mail address: cherniha@gmail.com 